\title{
Caesium and Lanthanum Suppression of Signal Fluctuation in Flame Atomic Absorption and Atomic Emission Spectrometry
}

\author{
Przemyslaw NiEDZIELSKI ${ }^{\dagger}$ and Armand DostatNI \\ Department of Analytical Chemistry, Faculty of Chemistry, Adam Mickiewicz University in Poznań, 89b \\ Umultowska Street, 61-614 Poznań, Poland
}

\begin{abstract}
This article describes the concept of controlling and correcting interference resulting from flame instability and sample reduction in absorption atomic spectrometry with flame atomization. Using popular laboratory spectrometers, which enable simultaneous or fast sequential measurements of many elements in the course of a single analysis, methods of controlling and correcting problems with instruments (e.g. reduction of sample flow) that directly affect the recorded analytical signal were elaborated.
\end{abstract}

Keywords Atomic absorption spectrometry, caesium, lanthanum, fast sequential analysis, signal correction

(Received March 20, 2014; Accepted May 7, 2014; Published July 10, 2014)

\section{Introduction}

The atomic absorption spectrometry (AAS) technique was invented and popularized in the 1950s. Despite the rapid development of new analytical tools (inductively induced plasma (ICP) and microwave induced plasma (MIP) spectrometry), this technique is still the most popular and preferred technique in scientific and commercial laboratories. ${ }^{1}$

Initially, the AAS technique was used in the single-element mode. However, new technologies enabled modifications in the analytical systems, which lead to the use of the AAS technique also in the multi-element mode., ${ }^{2,3}$ Several solutions were suggested, such as use of continuous radiation sources and, eventually, use of multi-channel devices, which are in use to this day. ${ }^{4-10}$ The solutions of multi-element determination in atomic absorption have been used in connection with the generation of volatile hydrides (hydride generation atomic absorption spectrometry: HGAAS) ${ }^{11}$, flame (flame atomic absorption spectrometry: FAAS) $)^{12}$ or electrothermal atomisation mode (electrothermal atomic absorption spectrometry: ETAAS). ${ }^{13-15}$ The flame atomic absorption spectrometry techniques also enable rapid sequential determination of elements whose resonance lines are in close proximity to one another. ${ }^{12,16,18} \mathrm{~A}$ few papers have presented the new application of multi-element determinations in atomic absorption spectrometry, namely interference correction using an internal standard. ${ }^{19-23}$ The literature discusses applications indicating the possibility of simultaneous determination of cadmium and lead ${ }^{9}$ or gold, cadmium, copper, nickel, lead, palladium, tin, and zinc using atomic absorption spectrometry with flame atomization. ${ }^{10}$

This article describes a procedure used to control and correct errors resulting from operation of flame atomic absorption spectrometers in the fast sequential mode (or in other

† To whom correspondence should be addressed.

E-mail: pnied@amu.edu.pl multi-element modes) using the FASS technique. The internal standard whose measurements were used to correct the interferences was the spectral buffer (Schinkel buffer), routinely used in the determinations of selected $(\mathrm{Ca}, \mathrm{Mg}, \mathrm{Na}, \mathrm{K})$ elements.

\section{Experimental}

\section{Reagents}

Only analytical grade reagents and deionised water produced in the Milli-Q device (Millipore, USA) were used. Determinations of metal content were performed using commercial analytical standards made by Merck (Merck, Germany), as well as $14 \mathrm{~mol} \mathrm{~L}^{-1}$ nitric acid and $10 \mathrm{~mol} \mathrm{~L}^{-1}$ hydrochloric acid (Merck, Germany). Moreover, Merck's Schinkel buffer was used in the atomic absorption spectrometry $\left(10 \mathrm{~g} \mathrm{~L}^{-1} \mathrm{CsCl}\right.$ and $\left.100 \mathrm{~g} \mathrm{~L}^{-1} \mathrm{La}\right)$. The LKSD-1 certified reference material (NRCan, Canada) that was used was a mixture of sediments from two lakes located in Ontario, Canada (approx. $40 \%$ of silicates and nearly $30 \%$ of organic matter).

\section{Instruments}

The metal content determinations were performed using the atomic absorption spectrometer SpectrAA 220FS (Varian, Australia), equipped with the SIPS-20 automatic system for standard solution preparation and on-line sample dilution (Varian, Australia). The following determination conditions were observed: a stoichiometric flame $\left(13.5 \mathrm{~L} \mathrm{~min}^{-1}\right.$ air and $2.0 \mathrm{~L} \mathrm{~min}^{-1}$ acetylene) was used in all determinations except for chromium determinations, where a flame with excess of inflammable gas was used $\left(11.5 \mathrm{~L} \mathrm{~min}^{-1}\right.$ air and $4.0 \mathrm{~L} \mathrm{~min}^{-1}$ acetylene).

The parameters measured in the determinations of particular elements are shown in Table 1. A calibration graph was prepared for each element using the spectrometer software, assuming a non-linear estimation function identified as the new rational, defined by the following formula: $C=A /\left(\mathrm{a} A^{2}+\mathrm{b} A+\right.$ 
Table 1 Characteristics of the methods of element determination by fast sequential atomic absorption and emission spectrometry $(n=6)$

\begin{tabular}{|c|c|c|c|c|}
\hline Element & $\begin{array}{c}\text { Equation of calibration } \\
C: \text { concentration, } A \text { : absorbance }\end{array}$ & $\begin{array}{c}\mathrm{CC}^{\mathrm{a}} \\
/ \mathrm{mg} \mathrm{L}^{-1}\end{array}$ & $\begin{array}{l}\text { Range } \\
/ \mathrm{mg} \mathrm{L}^{-1}\end{array}$ & $\begin{array}{l}\text { Precision }^{\mathrm{b}}, \\
\%\end{array}$ \\
\hline $\mathrm{Ca}$ & $C=A /\left(-0.01152 A^{2}+0.01240 A+0.02781\right)$ & 0.10 & 200 & 0.3 \\
\hline $\mathrm{Cd}$ & $C=A /\left(-0.18161 A^{2}+0.13425 A+0.35157\right)$ & 0.01 & 20 & 0.3 \\
\hline $\mathrm{Co}$ & $C=A /\left(-0.16087 A^{2}+0.02323 A+0.06898\right)$ & 0.06 & 20 & 1.1 \\
\hline $\mathrm{Cr}$ & $C=A /\left(-0.02875 A^{2}+0.00849 A+0.03823\right)$ & 0.08 & 20 & 1.5 \\
\hline $\mathrm{Cu}$ & $C=A /\left(-0.06596 A^{2}+0.01947 A+0.12367\right)$ & 0.04 & 20 & 0.8 \\
\hline $\mathrm{Fe}$ & $C=A /\left(-0.10967 A^{2}+0.02201 A+0.06347\right)$ & 0.07 & 50 & 1.1 \\
\hline $\mathrm{K}$ & $C=A /\left(-0.02962 A^{2}-0.00700 A+0.03558\right)$ & 0.10 & 100 & 0.2 \\
\hline $\mathrm{Mg}$ & $C=A /\left(-0.06829 A^{2}+0.03425 A+0.04255\right)$ & 0.10 & 50 & 1.2 \\
\hline $\mathrm{Mn}$ & $C=A /\left(-0.08781 A^{2}+0.05191 A+0.16472\right)$ & 0.03 & 20 & 0.6 \\
\hline $\mathrm{Na}$ & $C=A /\left(-0.02853 A^{2}-0.00838 A+0.03741\right)$ & 0.10 & 100 & 0.2 \\
\hline $\mathrm{Ni}$ & $C=A /\left(-0.12852 A^{2}+0.01825 A+0.07482\right)$ & 0.06 & 20 & 1.2 \\
\hline $\mathrm{Pb}$ & $C=A /\left(-0.07739 A^{2}+0.00441 A+0.03694\right)$ & 0.12 & 20 & 1.0 \\
\hline $\mathrm{Zn}$ & $C=A /\left(-0.29384 A^{2}+0.12238 A+0.15691\right)$ & 0.03 & 20 & 0.2 \\
\hline
\end{tabular}

a. Characteristic concentration. b. Concentration of $10 \%$ of range.

c), where $C$ is the concentration and $A$ the absorbance value. Moreover, the table lists the characteristic concentration (the concentration at which the value of the analytical signal (absorbance) obtained in the spectrometric measurement was equal to 0.0044), the scope of applicability of the calibration equation (the highest concentration at which the estimating function shown in Table 1 can be used), and the precision reflecting the distribution of the results for standard determinations at a concentration equal to $10 \%$ of the previously indicated modelling range. Standard solutions for calibration curves were prepared in the automatic spectrometer mode by way of automatic dilution of a multi-element basic solution using the SIPS-20 device. In routine determinations, the concentrations of particular metals were the same as the range of values shown in Table 1.

\section{Preparation of samples}

Extracts of lake sediment samples, including certified reference material, were prepared in compliance with the USEPA Method 3051A. A representative sample (1.20 g) was extracted with concentrated nitric acid and concentrated hydrochloric acid $(9.0 \mathrm{~mL}$ of nitric acid to $3.0 \mathrm{~mL}$ of hydrochloric acid) using microwave heating with a laboratory microwave unit.

\section{Results and Discussion}

\section{Methodology}

The spectrometer was factory-adjusted so as to enable working in the mode described by its manufacturer as fast sequential mode. In the spectrometer's optical system, radiation sources (HCL lamps) were installed in fixed positions. The radiation source was selected using a rotating mirror whose movement was coordinated with automatic selection of appropriate analytical wavelengths and slot value. When two or more lamps are switched on at the same time, fast (approx. $1-2 \mathrm{~s}$ ) retuning of the spectrometer's optical system is possible so as to perform sequential determinations of several elements in the same sample. ${ }^{19-22}$ Thus, it is possible to determine the content of several to several dozen elements in a single analysis and to combine the absorption and emission measurement modes, which significantly increases the analytical capabilities of the device.
The idea of correcting the changes in the analytical signal caused by external factors interfering with the determinations (flow of a sample, patency of the capillaries, change in the flame length, etc.) is directly based on the use of the options provided by the fast sequential mode. The subsequent measurements of the analytical signal (the analytical system's retuning time is equal to about $1 \mathrm{~s}$ ) for the individual elements enable the use of signal change correction tools similar to those used successfully in simultaneous or sequential measurements with the inductively or microwave-induced plasma techniques, which has not been possible due to the nature of the measurements in atomic absorption spectrometry.

Due to the possibility to perform sequential measurements of the analytical signal for many elements, measurement of an element or elements added in known and precisely specified quantities to all samples and standards appears to be advisable. Changes in the value of the analytical signal obtained as a result of the added value(s) provide information on interferences occurring in the analytical system. Moreover, information on the value of the analytical signal obtained for the element(s) added to the samples and for the standards may be used to correct these changes by appropriate recalculation of the analytical signal value for all the elements being determined.

The present study took advantage of the fact that caesium compounds and lanthanum compounds were added to samples in which the contents of sodium and potassium, as well as calcium and magnesium were being determined, respectively. This may be carried out by adding the off-the-shelf Schinkel buffer (containing both caesium and lanthanum compounds) to samples and standards, either manually or automatically (e.g. using the SIPS-20 device). Automatic dosing of the buffer is particularly suitable for obtaining highly precise caesium and lanthanum concentrations in the samples and standards. This enables using measurements of the analytical signal (in both the absorption and the emission mode; in this study measurements of radiation emission were used to measure the analytical signal for caesium and lanthanum) so as to correct signal changes for the other elements being determined.

\section{Use of caesium in signal change correction}

Figure 1A shows changes in the analytical signal during the analysis of a sample of acid soil extract. During the processes of aspiration, nebulisation, and then atomization, components of the sample are deposited in the capillary tube supplying the 


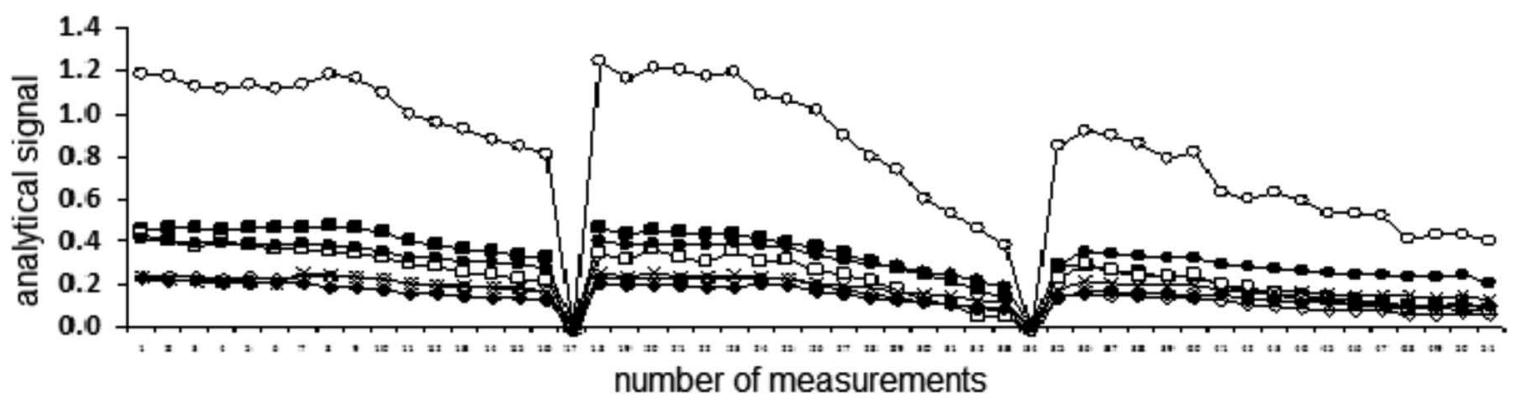

number of measurements

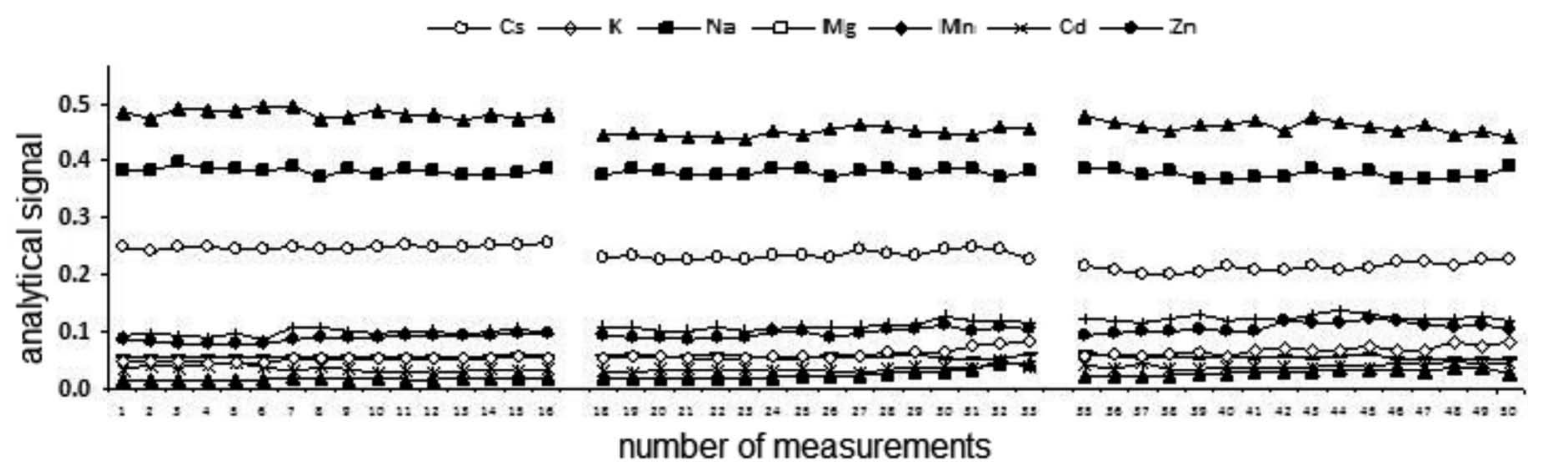

$\multimap-\mathrm{K} \rightarrow \mathrm{Na} \rightarrow-\mathrm{Mg} \rightarrow * \mathrm{Cr} \rightarrow-\mathrm{Cu} \longrightarrow \mathrm{Fe}-\mathrm{Co} \longrightarrow-\mathrm{Ni} \rightarrow \mathrm{Pb}$

Fig. 1 Analytical signal changes for caesium and selected elements before (A) and after (B) correction.

solution, as well as directly in the burner. If the analytical system is not systematically cleaned, the analytical signal value is reduced and, after approx. 20 analyses, a significant reduction of the length of the burner slot is observed. As a result, it is necessary to stop the analysis and thoroughly clean the analytical system. Inaccurate cleaning of the analytical system leads to a decrease in the quantity of material in the aspired sample or a reduction of the length of the optical route, with the resulting reduction of the strength of the recorded analytical signal, as shown in Fig. 1A.

The effects described herein occur with different intensity, depending on the sample matrix, often causing significant errors in the determinations. Because the signal for single elements are measured in AAS, so far, monitoring of the aforementioned effects consisted of analysing a control sample containing a known quantity of the element being determined (a standard, a reference material). The deviation of measured results from the real value indicated the presence of the effects in question. However, it was impossible to unequivocally identify the samples in whose analyses the problems with the analytical system did not occur. Thus, analyses had to be repeated for the entire series of samples (between analyses of control samples; usually several to several dozen samples).

In this study, the fast sequential technique described above was applied. However, this analytical interference correction method can be used in other techniques of atomic absorption spectrometry: ones where multi-channel spectrometers or continuous radiation sources are used.

This depends on the possibility to perform simultaneous or sequential determinations of many elements. By adding caesium to all standards and analysed samples, it was possible to identify analytical problems. Due to the constant and equal concentration of caesium in all the samples and standards, the analytical signal for this element should have a constant value in all analyses.
Assuming that the effects described here occur similarly in the case of all the elements being determined, changes in the analytical signal for caesium reflect fluctuations of the analytical signal for the other elements. Thus, it is possible to identify the decrease in the analytical signal related to the deposition of sample components in the capillary tube supplying the solution and directly in the burner. Moreover, computational compensation of changes in the analytical signal can be performed by comparing its value with the mean value of the analytical signal measured for caesium in the initial phase of the analysis (e.g. during the preparation of the calibration curve when there was no contamination in the analytical system), according to Eq. (1):

$$
s M_{\text {corrected }}=s M \cdot \frac{s C s}{s C s_{\text {calibration }}}
$$

where $s M_{\text {corrected }}$ is the analytical signal for the element being determined after correction, $s M$ the measured analytical signal for the element being determined, $s C s$ the measured analytical signal for caesium, $s C s_{\text {calibration the mean analytical signal for }}$ caesium obtained during calibration.

Signal correction enables obtaining a steady analytical signal within a wide range, regardless of the changes occurring in the analytical system, as shown in Fig. 1B. Even though interference in the analytical signal was high (Fig. 1A), the signal obtained after the correction was steady and matched the initial values (Fig. 1B). It is possible to identify problems with the analytical system not on the level of sample series analyses but rather for each single sample. Moreover, the identified interference in the analytical signal may be corrected in a broad range.

\section{Control using reference material}

In the tests of conformity of the results to the real value, the 
Table 2 Mean recovery for certified reference material LKSD-1 before and after caesium signal correction for selected elements $(n=9)$

\begin{tabular}{cccccccc}
\hline Recovery, $\%$ & $\mathrm{~K}$ & $\mathrm{Cr}$ & $\mathrm{Cu}$ & $\mathrm{Co}$ & $\mathrm{Ni}$ & $\mathrm{Cd}$ & $\mathrm{Pb}$ \\
\hline Before correction & $76.3 \pm 8.8$ & $67.5 \pm 11.2$ & $77.1 \pm 8.5$ & $75.5 \pm 7.7$ & $74.8 \pm 8.0$ & $77.0 \pm 8.4$ & $66.3 \pm 5.0$ \\
After correction & $95.1 \pm 1.4$ & $91.2 \pm 5.1$ & $96.1 \pm 1.9$ & $94.1 \pm 3.7$ & $93.8 \pm 3.0$ & $96.4 \pm 2.4$ & $93.2 \pm 3.1$ \\
\hline
\end{tabular}

A

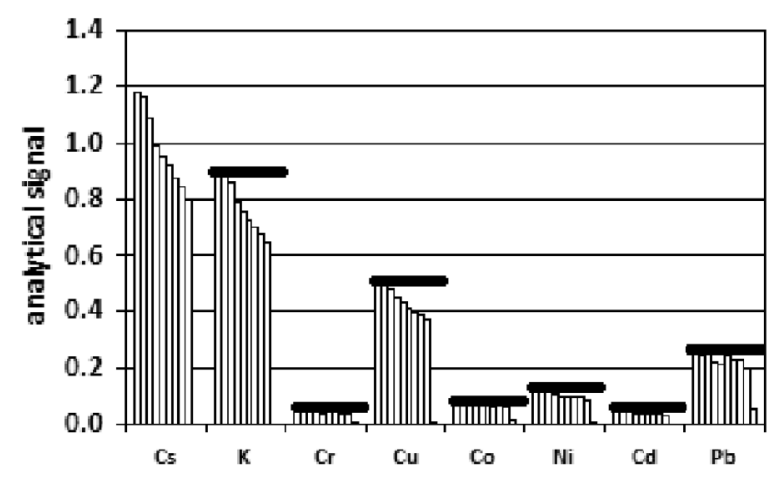

B

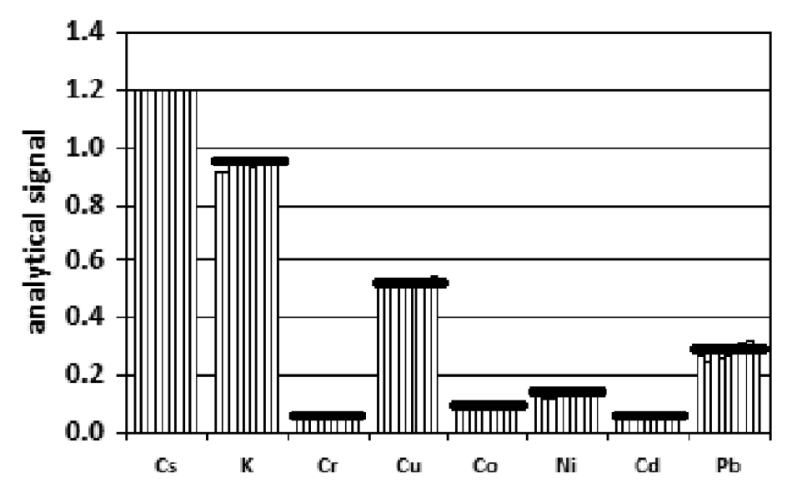

Fig. 2 Analytical signal for reference material extract analysis before (A) and after (B) caesium signal correction; the bold lines ilustrate the signal level corresponding to certified concentrations of the element being determined.

LKSD-1 certified reference material was used. In the reference material samples, the following seven elements were determined: $\mathrm{K}, \mathrm{Cr}, \mathrm{Cu}, \mathrm{Co}, \mathrm{Ni}, \mathrm{Cd}$, and $\mathrm{Pb}$. The analysis of each sample was repeated nine times during the series of analyses of other samples (the analysis of a reference material sample was repeated every 20 analyses of different samples). Due to the complex matrix of the analysed samples, effects connected with deposits of sample components were observed both in the capillary tube supplying the solution and in the burner, which directly affected the results of the analyses. This phenomenon caused a continuous decrease of the analytical signal and thus an increase in the differences between particular measurements.

On the other hand, these effects caused the mean recovery in a series of reference material analyses (Table 2, Fig. 2A) to reach approx. $70 \%$ with significant value of the RSD values were high at $5-11 \%$ for all elements being determined. The analytical signal change correction that was applied resulted in a significant increase in the mean recovery for a series of reference material analyses (Table 2. Fig. 2B), which exceeded
$90 \%$ for all the elements being determined, with significantly lower RSD values (below 5\%). This indicated the possibility and advisability of signal change correction in atomic absorption spectrometry.

\section{Use of lanthanum in signal change correction}

Due to the composition of the Schinkel buffer (which contains both caesium and lanthanum compounds that are necessary in the determinations of sodium and potassium, as well as calcium and magnesium, respectively) added to each sample during the analysis, it is possible, as with the caesium determination, to use the measurement of the analytical signal for caesium to identify and correct interferences in the analytical system.

The idea behind this is analogous to the use of analytical measurements for caesium. Moreover, it is possible and advisable to use the measurements performed for caesium and lanthanum in interference correction. Figure 3 shows simultaneous use of analytical signal corrections based on the signal measured for caesium and lanthanum. Figure 3A shows changes in the analytical signal for several selected elements caused by interferences in the analytical system: a reduction in the sample flow due to crystallization of components of a rich matrix sample and deposition of salts in the burner slot. A reduction of the value of the recorded analytical signal is noticeable, which prevents the measurement of the actual concentration of the element being measured in the sample.

The use of analytical signal measurements for caesium and lanthanum makes it possible to diagnose the aforementioned problems and to correct them based on the value of the analytical signal (Fig. 3B). The use of the signals measured both for caesium and lanthanum in the correction enables obtaining identical measurements, within a margin of uncertainty. The dual signal change correction system leads to an even greater independence from instrument-related factors affecting the changes in the analytical signal. ${ }^{19-22}$

\section{Conclusions}

The methods for correcting changes in analytical signals caused by instrument-related factors that are described herein enable a significant redefinition of analytical process control. ${ }^{19-22}$ They make it possible to switch from routine verifications of analyses of sample series to similar verifications performed for individual samples. Although they can be applied in custom-designed atomic absorption spectrometers, the growing popularity of these instruments makes it possible to plan the use of the methods described in the present article as a standard part of quality control procedures.

\section{Acknowledgements}

A. D. would like to thank the GT Projekt for content-related and financial support provided during his work on the doctoral thesis. The studies were partially financed by National Science 


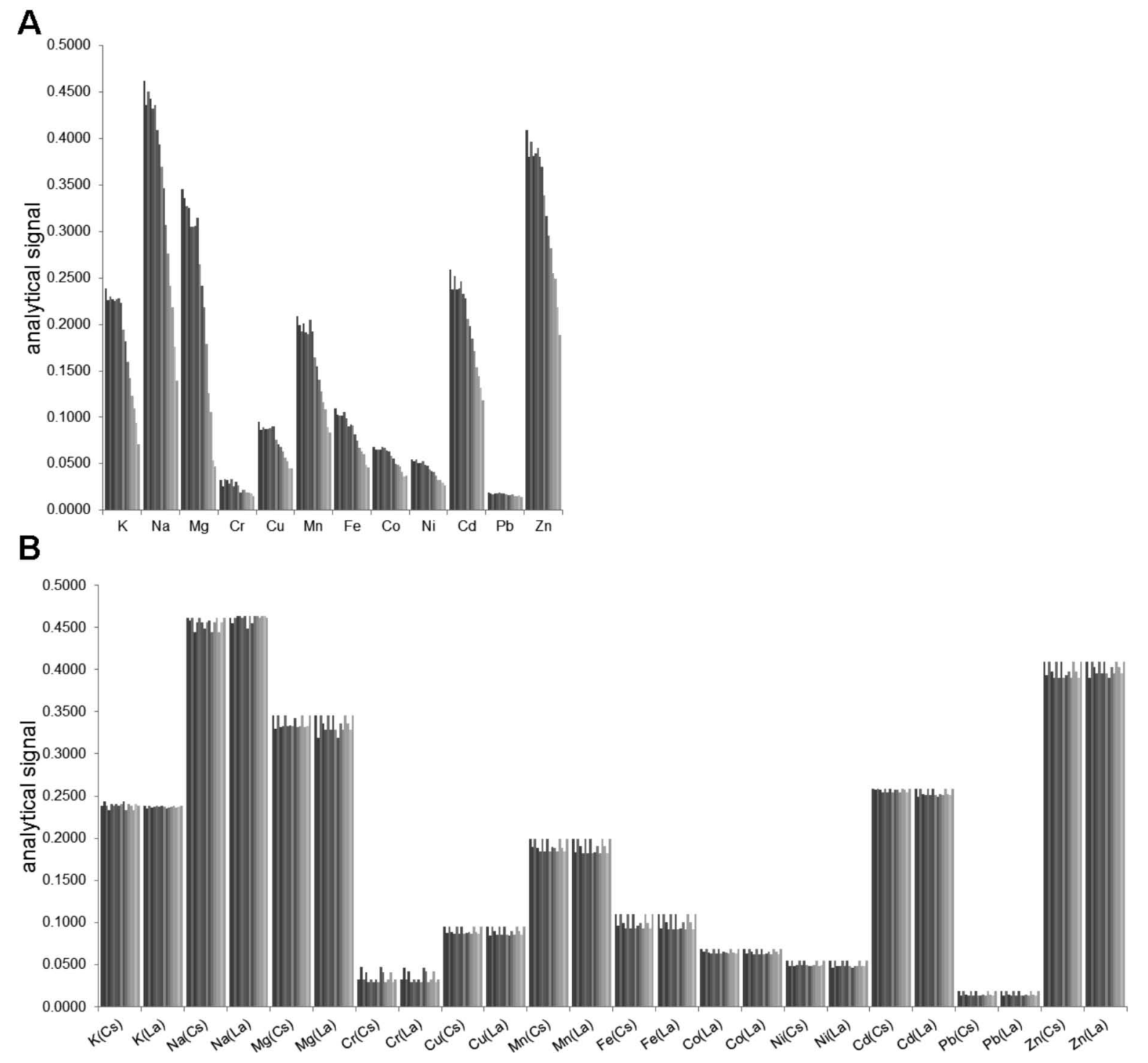

Fig. 3 Comparison of caesium and lanthanum signal correction $(n=16)$, A: signal before correction, B: caesium and lanthanum signal correction.

Centre Poland, based on the decision No. DEC-2013/09/B/ ST10/00610 and the described procedure will be used in the geochemical analysis.

\section{References}

1. N. H. Bings, A. Bogaerts, and J. A. C. Broekaert, Anal. Chem., 2013, 85, 670.

2. J. M. Harnly, T. C. O'Haver, B. Golden, and W. R. Wolf, Anal. Chem., 1979, 51, 2007.

3. J. M. Harnly, Anal. Chem., 1986, 58, 933A.

4. K. S. Farah and J. Sneddon, Appl. Spectrosc. Rev., 1995, 30, 351

5. B. Welz, D. L. G. Borges, F. G. Lepri, M. G. R. Vale, and U. Heitmann, Spectrochim. Acta, Part B, 2007, 62, 873.

6. J. M. Harnly, S. A. Lewis, and T. C. O’Haver, Anal. Chem., 1985, $57,2$.

7. L. M. G. dos Santos, R. G. O. Araujo, B. Welz, S. C. Jacobb, M. G. R. Vale, and H. Becker-Ross, Talanta, 2009, $78,577$.
8. F. A. C. Amorim and S. L. C. Ferreira, Talanta, 2005, 65 , 960.

9. A. Lindomar, H. S. Ferreira, W. N. L. dos Santos, and S. L. C. Ferreira, Microchem. J., 2007, 87, 77.

10. T. L. Thiem, Y. I. Lee, and J. Sneddon, Microchem. J., 1993, 48, 65.

11. M. B. B. Guerra, R. Carapelli, K. Miranda, A. R. A. Nogueira, and E. R. Pereira-Filho, Anal. Methods, 2011, 3, 599.

12. O. Sakurada, S. Tanaka, M. Taga, and T. Kakizaki, Analyst, 1991, 116, 31.

13. J. M. Harnly, Fresenius' J. Anal. Chem., 1996, 355, 501.

14. M.-C. Hsiang, Y.-H. Sung, and S.-D. Huang, Talanta, 2004, 62, 791 .

15. D. A. Katskov and Y. M. Sadagov, Spectrochim. Acta, Part $B$, 2011, 66, 451 .

16. S. R. Oliveira, J. L. Raposo Jr., and J. A. G. Neto, Spectrochim. Acta, Part B, 2009, 64, 593.

17. E. G. P. da Silva, V. Hatje, W. N. L. dos Santos, L. M. Costa, A. R. A. Nogueira, and S. L. C. Ferreira, J. Food Compos. Anal., 2008, 21, 259. 
18. B. Welz, S. Morés, E. Carasek, M. G. R. Valeb, M. Okruss, and H. Becker-Rossd, Appl. Spectrosc. Rev., 2010, 45, 327.

19. S. L. C. Ferreira, E. G. P. da Silva, L. A. Portugal, G. D. Matos, F. A. de Santana, M. G. A. Korn, and A. C. S. Costa, Anal. Lett., 2008, 41, 1571.

20. K. Miranda, A. G. G. Dionísio, and E. R. Pereira-Filho, Microchem. J., 2010, 96, 99.
21. S. L. C. Ferreira, A. S. Souza, G. C. Brandao, H. S. Ferreira, W. N. L. dos Santos, M. F. Pimentel, and M. G. R. Vale, Talanta, 2008, 74, 699.

22. H. D. Projahn, U. Steeg, J. Sanders, and E. Vanclay, Anal. Bioanal. Chem., 2004, 378, 1083.

23. U. Heitmann, H. Becker-Ross, and D. Katskov, Spectrochim. Acta, Part B, 2006, 61, 351. 